\title{
HIGHLIGHTS
}

DIARRHEA

\section{Impaired feedback inhibition of bile acid synthesis associated with bile acid diarrhea}

Primary bile acid malabsorption (BAM) accounts for $\sim 30 \%$ of functional chronic diarrhea cases. Julian Walters and colleagues now report that reduced feedback inhibition of hepatic bile acid synthesis in the ileum may contribute to the pathogenesis of BAM due to unregulated bile acid production that exceeds the ileal resorptive capacity. "The mouse ileal hormone, fibroblast growth factor 15 (Fgf15) regulates feedback inhibition of bile acid synthesis," explains Walters, "and we postulated that deficiency of its human ortholog FGF-19 might cause the human disease."

The investigators measured fasting serum levels of the bile acid precursor 7a-hydroxy-4-cholesten-3-one (C4)-a measure of bile acid synthesis-in 17 patients with BAM and found them to be significantly higher than in 19 healthy individuals (median $51 \mathrm{ng} / \mathrm{ml}$ versus
$18 \mathrm{ng} / \mathrm{ml}$, respectively). Conversely, patients with BAM had decreased concentrations of FGF-19 compared with healthy participants (median $120 \mathrm{pg} / \mathrm{ml}$ versus $231 \mathrm{pg} / \mathrm{ml}$ ). Correlation analysis of the data revealed that levels of FGF-19 were inversely related with those of $\mathrm{C} 4$ in individuals with BAM, but not in healthy controls.

\section{4 ...patients with BAM had} decreased ... FGF-19 compared with healthy participants 77

Patients with low selenium homocholic acid taurine (SeHCAT) levels-indicative of abnormal bile acid retention-and no cholecystectomy (type 2 BAM) all had significantly lower FGF-19 values than healthy participants. Furthermore, significantly increased C4 and decreased
FGF-19 concentrations were observed in individuals who had diarrhea due to BAM secondary to previous ileal surgery (type 1 BAM).

The researchers also identified a fasting level of FGF-19 $\leq 146 \mathrm{pg} / \mathrm{ml}$ as a potential marker for BAM that had a diagnostic performance similar to that of C4 levels. They suggest that confirmation of these findings may lead to the development of FGF-19-specific diagnostic tests for primary BAM. Moreover, the investigators next plan to identify the exact ileal defect that leads to the impaired FGF-19 release and explore its potential as a target for novel drug therapy.

Rowan Higgs

Original article Walters, J. R. F. et al. A new mechanism for bile acid diarrhea: defective feedback inhibition of bile acid synthesis. Clin. Gastroenterol. Hepatol. 7, 1189-1194 (2009) 